\title{
PREDICTION OF DRIVING ABILITY IN PEOPLE WITH DEMENTIA- AND NON-DEMENTIA-RELATED BRAIN DISORDERS
}

\author{
Carrie Innes ${ }^{1,2}$, Richard Jones ${ }_{5}^{1,2,3}$, John Dalrymple-Alford ${ }^{1,4}$, \\ Julie Severinsen ${ }^{5}$, and Jane Gray ${ }^{5}$ \\ ${ }^{1}$ Van der Veer Institute for Parkinson's \& Brain Research \\ ${ }^{2}$ Dept Medical Physics \& Bioengineering, Christchurch Hospital \\ ${ }^{3}$ Dept Medicine, University of Otago, Christchurch \\ ${ }^{4}$ Dept Psychology, University of Canterbury \\ ${ }^{5}$ Dept Occupational Therapy, Burwood Hospital \\ Christchurch, New Zealand \\ Email: carrie.innes@vanderveer.org.nz
}

\begin{abstract}
Summary: Brain disorders can impair physical and cognitive functions necessary for safe driving. Two hundred people with brain disorders referred for a driving assessment were recruited and their performance on a computerized battery of sensory-motor and cognitive tests (SMCTests) and a blinded on-road assessment determined. Based on SMCTests performance, binary logistic regression (BLR) and nonlinear causal resource analysis (NCRA) models classified on-road pass or fail with $70 \%$ accuracy. Greater accuracy could be achieved by splitting referrals into two groups: (1) Dementia and (2) Non-dementia-related brain disorders. BLR models classified on-road driving outcome as pass or fail with accuracies of $76 \%$ (Dementia) and 75\% (Non-dementia), while NCRA models had accuracies of $77 \%$ (Dementia) and 80\% (Non-dementia). Measures of attention were most critical for predicting driving ability in the dementia group. In the non-dementia group, prediction of driving ability was most accurate with assessment of a broader range of sensory-motor and cognitive functions. Compared to BLR, NCRA was able to identify and use additional measures to improve accuracy. NCRA is also better able to accommodate outliers due to it being a non-linear modelling method based upon individual performance-limiting impairments. We propose three main factors underlying sub-optimal prediction of driving ability based on SMCTests performance: (1) there are one or more functions important for driving ability which are not currently assessed with SMCTests - these could be sensory-motor or cognitive or other (e.g., attitude, confidence, insight, road code knowledge); (2) suboptimal classification/prediction techniques or models; or (3) inaccuracies in the on-road driving assessments.
\end{abstract}

\section{BACKGROUND}

A computerized battery of sensory-motor and cognitive tests (SMCTests) has been developed comprising tests of visuoperception, visuomotor ability, complex attention, visual search, decision-making, impulse control, planning, and divided attention. A previous study with persons with brain disorders $(\mathrm{n}=50)$ has shown that driving ability can be predicted using a subset of SMCTests (Innes et al., 2007). The current study aimed to validate prediction with a larger group of referrals to Burwood Hospital's Driving and Vehicle Assessment Service (DAVAS). An accurate estimation of driving ability will minimize on-road assessments of 
patients who will inevitably fail, thus decreasing the unnecessary risk of accident these entail. SMCTests is also designed to identify physical, perceptual, and cognitive deficits underlying an inability to drive safely so that focussed rehabilitation might lead to safe driving in some cases.

\section{OBJECTIVES}

The objective of the study was to obtain performance data on SMCTests from a group of 200 DAVAS referrals to improve the on-road predictive model equations established in our earlier study of $n=50$ DAVAS referrals. The study also aimed to determine the predictive value of SMCTests with different neurological disorders, including declining brain function with old age, and to determine which neurological deficits are more critical for driving.

\section{METHODS}

\section{Participants}

Ethical approval for this study was obtained from the Upper South A Regional Ethics Committee. To be eligible for inclusion in the study, referrals had to have a definite or suspected brain disorder due to traumatic brain injury, stroke, tumour, neurological degeneration, or age-related substantive cognitive decline, but must have been free from any unrelated diagnosed psychiatric illness. Referrals either held a current full driver's licence or had held a full driver's licence prior to brain injury, onset of disease, or cognitive decline. $\mathrm{N}=200$ DAVAS referrals ( $n=143$ males, $n=57$ females) were recruited. Study referrals had a mean age of 70.8 years (range 19-92 years). Diagnostic information is included in Table 1.

Table 1. Diagnoses of DAVAS study referrals

\begin{tabular}{lc}
\hline Diagnosis & $\mathrm{n}$ \\
\hline Diagnosed or suspected early dementia & 95 \\
Stroke & 62 \\
Traumatic brain injury & 21 \\
Parkinson's disease & 8 \\
Hypoxic brain injury / Transient ischaemic attack & 7 \\
Brain tumour & 3 \\
Other neurological disorder & 4 \\
\hline
\end{tabular}

\section{Apparatus}

The off-road assessment equipment included a laptop (incorporating the SMCTests software) and screen for the assessor, and a separate screen, steering wheel, and set of accelerator and brake pedals for the participant. Figure 1 shows the set-up for the off-road assessment apparatus.

\section{Tests}

SMCTests comprises three visuoperception tests, four non-tracking visuomotor tests, three tracking visuomotor tests, and cognitive function tests of complex attention, divided attention, visual search, decision-making, impulse control, and planning. Baseline performance data for SMCTests has been established with a study involving 60 healthy control subjects, comprising 30 females and 30 males and a mean age of 50.2 years (range 22-78 years) (Innes et al., in press). 
A subset of SMCTests, established by the predictive modelling techniques in the initial study, was used in the study. These tests are fully computerized and the responses have been designed to be contextually related to on-road driving such as responding to the presentation of a red light by pressing the brake or turning the steering wheel in order to maintain steering accuracy. Through this design technique referrals can be completely computer-naïve and, yet, not disadvantaged on any of the tests.

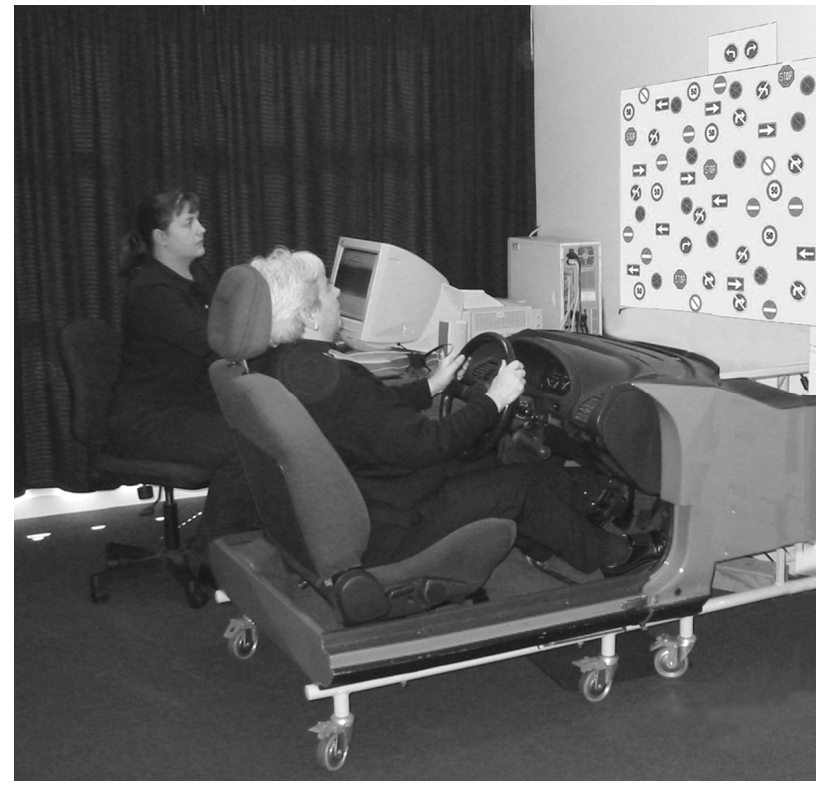

Figure 1. The apparatus used during the off-road assessment

The subset of tests comprised four sensory-motor tests and three cognitive tests and are described in detail elsewhere (Heitger et al., 2004; Jones, 2006; Innes et al., 2007; Innes et al., in press). Footbrake Reaction measures the reaction and movement times at which a subject can respond to an unexpected signal by moving his or her foot from the accelerator to the brake. Ballistic Movement measures the reaction time and maximum speed at which a subject can turn the steering wheel to move an arrow out of a box and across a pass-line in response to an unexpected signal. Tracking measures the accuracy with which a subject can track a laterally moving target using the steering wheel to move an on-screen arrow. The tracking target may move in a sine wave (Sine Tracking) or random wave (Random Tracking) (Figure 2). Complex Attention assesses a subject's ability to sustain attention over an extended period of time. Subjects must turn the steering wheel from left to right repeatedly to maintain an arrow in a box on the same side of the screen as a green light symbol is being presented. Variability in reaction times are analysed to identify lapses in concentration. Divided Attention assesses ability to divide attention between two simultaneously-performed separate activities. Random Tracking is combined with a simultaneous visual scanning task. While the subject tracks the random target, consecutive sets of four arrows are displayed. The subject has to maintain accurate tracking of the target, while determining whether the arrows are pointing in the same direction or not. Planning assesses ability to use accurate timing and judgement as an indicator of planning ability. The subject is presented with a screen showing a bird's eye view of a road and surrounds. When the subject presses the accelerator, the road and surrounds scroll down the screen. The blue car must drive as far as possible in 6 min while avoiding all hazards. 


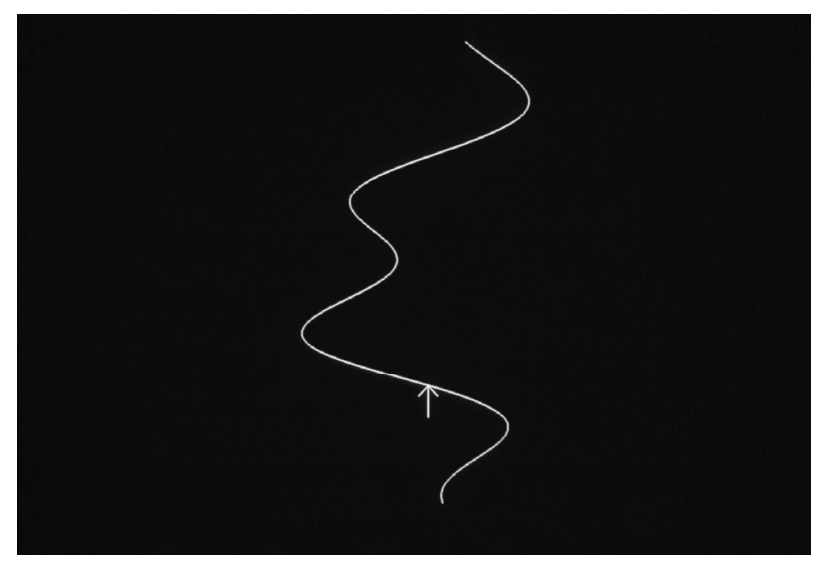

Figure 2. A snap-shot of the Preview Random Tracking test

\section{On-Road Assessment}

An on-road assessment provided the criterion measure ('gold standard') of driving ability. Performance during the on-road assessment was evaluated by an occupational therapist and an independent driving instructor, both blinded to performance on SMCTests. The driving instructor, who has experience in driver assessment of persons with disabilities and/or brain disorders, was seated in the front passenger seat. He was responsible for giving directions to the subject and for maintaining the safety of the vehicle. The occupational therapist, experienced in driving assessment and rehabilitation of persons with brain disorders and/or disabilities, was seated in the rear of the car.

All assessments began on the hospital grounds where the subject's ability to control the initial starting and stopping of the vehicle was assessed. Subjects were then asked to drive to a suburb immediately adjacent to the Hospital. This suburb is residential and experiences little traffic during the day but includes controlled (give-way and stop-sign controlled) and uncontrolled intersections. Subjects were then asked to drive in increasingly busy and complicated traffic situations. Traffic hazards included single-lane roundabouts, dual-lane roundabouts, dual-lane roads, controlled intersections (give-way, stop sign, and traffic light controlled) uncontrolled intersections, and changes in speed zone (i.e., $50 \mathrm{~km} / \mathrm{hr}, 60 \mathrm{~km} / \mathrm{hr}$, and $80 \mathrm{~km} / \mathrm{hr}$ sections). Assessments were approximately $45 \mathrm{~min}$ in duration. However, if the occupational therapist or driving instructor considered that their safety or the safety of the vehicle or other road users was at risk at any stage during the assessment, the assessment was terminated and the driving instructor drove the vehicle back to the Hospital.

On-road driving performance was scored by the occupational therapist as a Pass or a Fail using the Advanced Driving Assessment System. Assessment was defined by four areas of driving deemed necessary for safe and able driving: search, hazard identification, controls, and observation of traffic regulations, each subdivided into specific components. The occupational therapist also used a driving scale to evaluate driving performance (outlined in Innes et al., 2007). A Driving Score was determined by mutual agreement by the two assessors. If a subject was given a Driving Score between 0-5, they were considered to have failed the on-road assessment. A Driving Score between 6-10 indicated a pass. 


\section{Data Analysis}

Binary logistic regression (BLR) analysis was used to determine the predictive value of SMCTests at the individual level. Other non-SMCTests measures such as dementia status, sex, and age were not used as potential predictor variables. This form of regression is used when the dependent variable is dichotomous (i.e., pass or fail) and allows prediction of outcome from a set of variables that may be continuous, ordinal, dichotomous, or a mix.

Nonlinear causal resource analysis (NCRA) was also used to determine the predictive value of SMCTests at the individual level. As with BLR, only SMCTests measures were used as potential predictor variables. NCRA is a relatively new approach to performance prediction and is based on the resource economic performance modelling constructs of General Systems Performance Theory and the Elemental Resource Model (Kondraske, 1995). With NCRA, the minimum resource level required to achieve a given level of performance on a given high-level task is determined for each resource involved in the task (Fischer et al., 2002; Kondraske, 2006). Resource demand functions are calculated which can then be used to predict performance in an independent subject.

\section{RESULTS}

Of the total group, $n=88(44 \%)$ passed and $n=112(56 \%)$ failed the on-road assessment. The on-road pass group was younger (mean age $64.4 \mathrm{yr}$ ) than the on-road fail group (mean age $75.8 \mathrm{yr})$ (t-test $p<.001)$.

Both BLR and NCRA models were $70 \%$ accurate in classifying on-road pass or fail based on SMCTests performance (see Table 2), but differed in their sensitivities and specificities. Compared with the NCRA model, the BLR model had greater sensitivity for correctly classifying a referral as an on-road fail (sensitivity: BLR 74\%, NCRA 46\%). However, the NCRA model had greater specificity for correctly classifying a referral as on-road pass (specificity: NCRA $99 \%$, BLR 64\%).

Table 2. Classification model results for the entire referral group $(n=200)$

\begin{tabular}{lcccc}
\hline Model & Accuracy & Sensitivity & Specificity & $\begin{array}{c}\text { ROC - Area } \\
\text { under the Curve }\end{array}$ \\
\hline BLR & $70 \%$ & $74 \%$ & $64 \%$ & 0.783 \\
NCRA & $70 \%$ & $46 \%$ & $99 \%$ & 0.800 \\
\hline
\end{tabular}

Measures from Divided Attention and Complex Attention were identified by the BLR model as useful for classifying on-road driving outcome as pass or fail. The NCRA model classification utilized the same measures plus measures of planning, visuomotor tracking, and upper- and lower-limb ballistic movement.

Referrals were then split into two groups: (1) Dementia (diagnosed or suspected early dementia as determined by the referring source, $n=95$ ) and (2) Non-dementia (all other brain disorders, $\mathrm{n}=105)$. The Dementia group comprised $68 \%$ males $(\mathrm{n}=65$ males, $\mathrm{n}=30$ females $)$ and $59 \%$ fails $(\mathrm{n}=56$ fails, $\mathrm{n}=39$ passes $)$. The Non-dementia group comprised $74 \%$ males $(\mathrm{n}=78$ males, $\mathrm{n}=27$ females) and $53 \%$ fails ( $\mathrm{n}=56$ fails, $\mathrm{n}=49$ passes). 
Model classification accuracy, sensitivity, and specificity for the Dementia and Non-dementia groups are shown in Table 3. Compared with models for the entire referral group, more accurate classification of on-road driving outcome could be achieved by splitting the referrals into the two diagnosis groups (model accuracies: Dementia group 76-77\%, Non-dementia group 75-80\%). NCRA models produced more accurate classifications of on-road driving outcome than BLR (model accuracies: NCRA 77-80\%, BLR 75-76\%). NCRA models also had greater specificity for correctly classifying a referral as on-road pass (specificity: NCRA 95-98\%, BLR 72-76\%). However, as for the entire referral group, BLR models had greater sensitivity for correctly classifying referrals as on-road fail than NCRA (sensitivity: BLR 74-79\%, NCRA 64\%).

The BLR model identified measures of divided attention and complex attention as predictive of on-road driving in the dementia group, whereas the NCRA model identified the same measures with the addition of planning and visuomotor tracking measures. In the non-dementia group, the BLR model identified measures of upper-limb ballistic movement, complex attention, and planning as predictive of on-road driving, whereas the NCRA model identified the same measures plus measures of divided attention and visuomotor tracking.

Table 3. Classification model results for the Dementia $(n=95)$ and Non-dementia $(n=105)$ groups

\begin{tabular}{llcccc}
\hline Group & Model & Accuracy & Sensitivity & Specificity & $\begin{array}{c}\text { ROC - Area } \\
\text { under the Curve }\end{array}$ \\
\hline \multirow{2}{*}{ Dementia } & BLR & $76 \%$ & $79 \%$ & $72 \%$ & 0.813 \\
& NCRA & $77 \%$ & $64 \%$ & $95 \%$ & 0.822 \\
\multirow{2}{*}{ Non-dementia } & BLR & $75 \%$ & $75 \%$ & $76 \%$ & 0.845 \\
& NCRA & $80 \%$ & $64 \%$ & $98 \%$ & 0.877 \\
\hline
\end{tabular}

\section{DISCUSSION}

For both the dementia and non-dementia groups, NCRA identified the same measures as useful for correctly classifying on road driving outcome as BLR but was able to identify and use additional measures to improve accuracy. NCRA is better able to accommodate outliers due to it being a non-linear modelling method based upon individual performance-limiting impairments. Measures of attention (complex and divided) were important for predicting on-road driving in both diagnosis groups but were most critical in the dementia group. In contrast, predictive models which include a broader assessment of sensory-motor, cognitive, and coordinated sensory-motor and cognitive functions are more accurate for predicting on-road driving in people with other brain disorders such as stroke and traumatic brain injury.

SMCTests provides useful data regarding sensory-motor and cognitive dysfunction in people with brain disorders and is considered a crucial part of the driving assessment by the three occupational therapy teams who use SMCTests in New Zealand. In comparison to other driving assessment tests batteries, SMCTests provides a more comprehensive assessment of sensorymotor and cognitive function and a similar or more accurate prediction of on-road driving ability (Galski et al., 1993; Dobbs, 1997; National Highway Traffic Safety Administration, 1999; Fischer et al., 2002). However, to eliminate the need for unnecessary on-road driving assessments the predictive accuracy of an off-road battery needs to be substantially higher than we or others in the literature have been able to achieve. Thus, currently, on-road assessment is still required to make a final decision regarding on-road driving safety. 
We propose three main factors underlying sub-optimal prediction of driving ability based on SMCTests performance: (1) there are one or more functions important for driving ability which are not currently assessed with SMCTests - these could be sensory-motor or cognitive or other (e.g., attitude, confidence, insight, road code knowledge); (2) suboptimal classification or prediction techniques or models; or (3) inaccuracies in the on-road driving assessments.

\section{ACKNOWLEDGEMENTS}

The authors would like to acknowledge funding for this project from the Road Safety Trust and a Canterbury Medical Research Foundation Fellowship.

\section{REFERENCES}

Dobbs, A. R. (1997). Evaluations for at-risk experienced drivers. Edmonton, Canada: DriveABLE Testing, Ltd.

Fischer, C. A., Kondraske, G. V., \& Stewart, R. M. (2002). Prediction of driving performance using nonlinear causal resource analysis. Proceedings of the Second Joint Eng Med Biol Soc/Biomed Eng Soc Conference, 2, 2473-2474.

Galski, T., Bruno, R. L., \& Ehle, H. T. (1993). Prediction of behind-the-wheel driving performance in patients with cerebral brain damage: a discriminant function analysis. American Journal of Occupational Therapy, 47, 391-396.

Heitger, M. H., Anderson, T. J., Jones, R. D., Dalrymple-Alford, J. C., Frampton, C. M., \& Ardagh, M. W. (2004). Eye movement and visuomotor arm movement deficits following mild closed head injury. Brain, 127, 575-590.

Innes, C. R. H., Jones, R. D., Anderson, T. J., Hollobon, S. G., \& Dalrymple-Alford, J. C. (in press). Performance in normal subjects on a novel battery of driving-related sensory-motor and cognitive tests. Behavior Research Methods.

Innes, C. R. H., Jones, R. D., Dalrymple-Alford, J. C., Hayes, S., Hollobon, S., Severinsen, J., Smith, G., Nicholls, A., \& Anderson, T. J. (2007). Sensory-motor and cognitive tests predict driving ability of persons with brain disorders. Journal of the Neurological Sciences, 260, 188-198.

Jones, R. D. (2006). Measurement of sensory-motor control performance capacities: Tracking tasks. In J. D. Bronzino (Ed.), The Biomedical Engineering Handbook - Biomedical Engineering Fundamentals (3rd ed., Vol. 1, pp. 77:71-25). Boca Raton, Florida: CRC Press.

Kondraske, G. V. (1995). An elemental resource model for the human-task interface. International Journal of Technology Assessment in Health Care, 11, 153-173.

Kondraske, G. V. (2006). Chapter 75. The elemental resource model for human performance. In J. D. Bronzino (Ed.), The Biomedical Engineering Handbook - Biomedical Engineering Fundamentals (3rd ed., pp. 1 - 19). Boca Raton, Florida: CRC Press.

National Highway Traffic Safety Administration. (1999). Safe mobility for older people notebook (DOT HS 808853). Washington, DC: US Department of Transportation. 